This item was submitted to Loughborough's Research Repository by the author.

Items in Figshare are protected by copyright, with all rights reserved, unless otherwise indicated.

\title{
Comparison between CTIA hand phantom and different human hands for OTA power measurements
}

PLEASE CITE THE PUBLISHED VERSION

http://dx.doi.org/10.1109/APS.2012.6348026

PUBLISHER

(C) IEEE

VERSION

AM (Accepted Manuscript)

LICENCE

CC BY-NC-ND 4.0

\section{REPOSITORY RECORD}

Ojerinde, Oluwaseun A., C.J. Panagamuwa, W.G. Whittow, and R.M. Edwards. 2019. "Comparison Between CTIA Hand Phantom and Different Human Hands for OTA Power Measurements". figshare.

https://hdl.handle.net/2134/11663. 


\title{
Comparison Between CTIA Hand Phantom and Different Human Hands for OTA Power Measurements
}

\author{
O. A. Ojerinde, C. J. Panagamuwa, W. G. Whittow and R.M. Edwards \\ School of Electronic, Electrical and Systems Engineering \\ Loughborough University \\ Loughborough, UK \\ O.A.Ojerinde@lboro.ac.uk, C.J.Panagamuwa@lboro.ac.uk,W.G.Whittow@lboro.ac.uk, R.M.Edwards@lboro.ac.uk
}

\begin{abstract}
This study investigates the Over the Air (OTA) power measurements from a mobile phone when held by the CTIA hand phantom compared to a number of participant's hands. A measurement setup in a shielded environment was setup to ensure that all the participants held the mobile phone in a similar fashion to the hand phantom so that accurate comparisons could be made. Measured results show that the received power from all the participants was lower than that received from the hand phantom.
\end{abstract}

\section{INTRODUCTION}

It has long been known that the hand holding the mobile phone during a call can have a detrimental effect on the power received at the base station [1]. Depending on the position of the hand (and the fingers) and the mobile phone antenna, the Over the Air (OTA) performance could decrease by over 10dB. Recently, the Cellular Telecommunications Industry Association (CTIA) has recommended the use of a hand phantom when carrying out OTA measurements [2]. A number of companies have now developed solid hand phantoms with different grip styles to accommodate varying mobile phone types. The ICES TC34 SC2 working group is also currently working on assessing the feasibility of including this hand phantom in the Specific Absorption Rate (SAR) measurement standard. The CTIA hand phantom is thus gaining increased prominence in today's mobile communications field.

Kuster et al. in [3] investigated the effects of the hand on OTA performance based on a simulation study. The study found that the position of the index finger was the most critical parameter. However, other parameters such as phone to palm separation distance, other finger positions and hand size were all found to have effects. In [4], a hand phantom was used to investigate efficiency, antenna match and total radiated power (TRP). The work showed that certain hand grip positions could increase the TRP at the low bands and reduce the TRP at high bands. It was concluded that this was due to the ability of the hand phantom to extend the ground plane. No actual human hands were used in either study.
The paper presented here, investigates the effects real human hands and the CTIA hand phantom on mobile phone transmission performance. As the hand phantom increases its penetration into the communications field, this study aims to establish how representative its results are compared to human hands of varying size.

\section{EXPERIMENTAL DESCRIPTION}

The experiment was setup in one of the anechoic chambers at Loughborough University. The experimental setup is shown in Fig. 1.

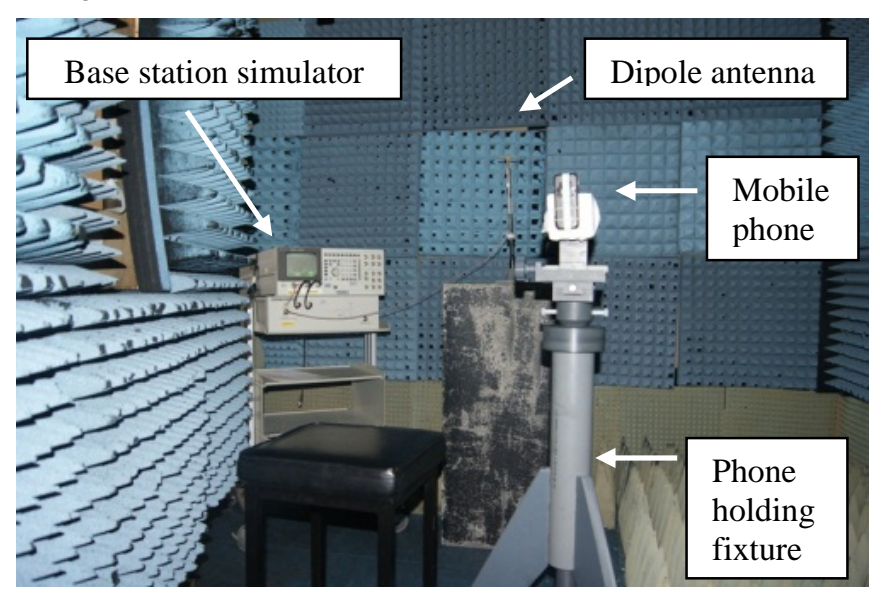

Figure 1. Measurement setup in the anechoic chamber

A Nokia 6220c was used for this experiment because its antenna was located at the top of the phone. The location of the antenna can be seen in Fig. 2. The phone was positioned at a fixed location using high density foam and two thin cableties. An Agilent 8922M was used to simulate the base station which used a dipole antenna to communicate with the mobile phone. The base station instructed the phone to transmit maximum power in one of the GSM1800 low bands. The power transmitted by the phone was measured using a spectrum analyzer connected to a horn antenna pointing 
directly at the phone. The phone was placed with its back facing towards the horn antenna. The phone - horn antenna separation distance was kept constant. Participants were asked to sit to the left of the mobile phone and to grip the phone with their right hand. The stool on which the participants sat was kept in the same location to ensure the participant's bodies were in the same position with respect to the phone. Seven participants took part in the study.

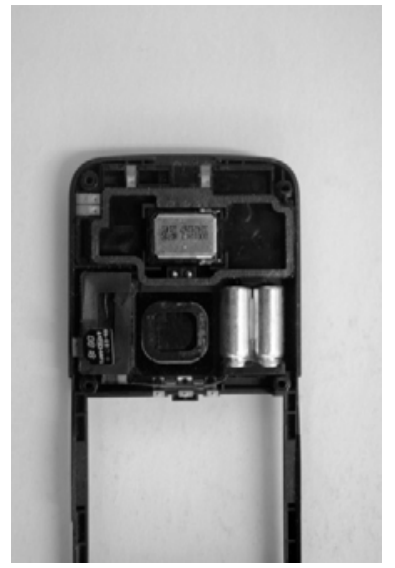

Figure 2. Antenna location of the Nokia 6220c

The index finger positions were carefully controlled using markers on the back of the phone. Paper tape was fixed vertically along the centre of the back and marked with four $10 \mathrm{~mm}$ spacings. The top most position (Position 1) was directly over the phone antenna and Position 4 was directly $40 \mathrm{~mm}$ below this. The Participants were asked to hold the phone in a similar fashion to the hand phantom.

\section{RESULTS}

In order to normalize the measured results, a measurement was taken with the mobile phone in the correct position but without a hand. The received maximum power received at the horn antenna at the transmit frequency was $-4.37 \mathrm{dBm}$. All results presented hereon are normalized to this value.

Received power results were then recorded for the CTIA phantom hand in the four different positions. The process was conducted once more in order to check the repeatability of the measurement. The normalized results are shown in Fig. 3. Although there was very good agreement in two of the positions, the maximum error between the two sets of measurements was $1 \mathrm{~dB}$. This corresponded to Position 4 where a major part of the phantom hand was not in contact with the phone and the spacer which normally fixed the separation distance between the phone and the palm became obsolete.

The normalized measured results for hands 1 to 7 are given in Fig. 3. As expected for all participants, as the index finger moves away from the antenna, the received power at the horn antenna increases. In two cases the hand seems to increase the received power beyond that received when the phone alone was measured. In the case of participant 4 , there is a $2 \mathrm{~dB}$ increase in the received power. In these cases the hand is most likely interacting with the mobile phone's radiation pattern and causing more radiation to be redistributed towards the horn antenna.

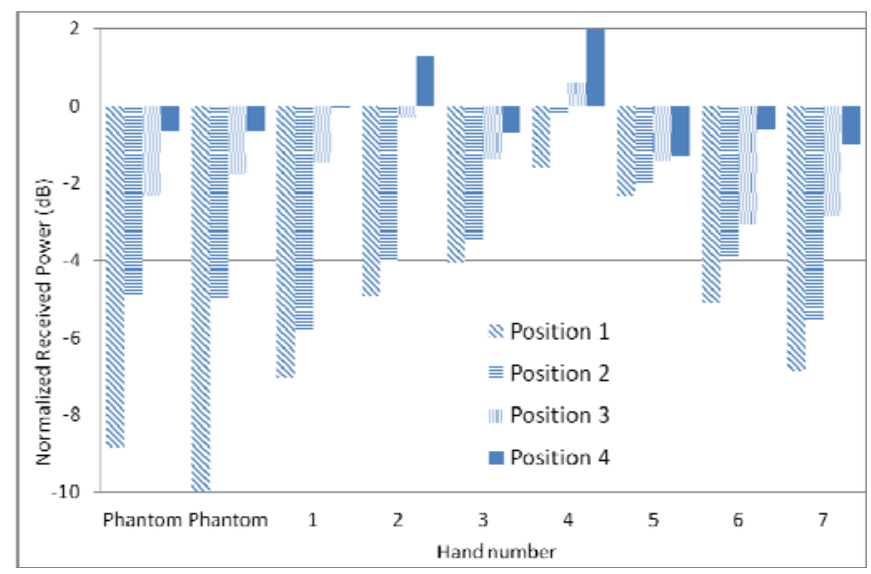

Figure 3. Normaized received power values for different hands in four positions

The results also indicate that in Position 1the phantom hand causes a larger attenuation in the power received by the horn antenna compared to all 7 participant's hands. In the other three hand positions, the results seem more random. The authors intend to measure the dimensions and the volume of the participant's hands and investigate if there is a relationship between the size of the hand, index finger size and width etc. and the level of attenuation.

\section{CONCLUSIONS}

This initial study comparing the CTIA hand phantom to actual human hands suggests that the phantom hand will tend to overestimate the attenuation in OTA performance measurements when the index finger covers the antenna. Many more participants are needed to make this study representative of the general public.

\section{ACKNOWLEDGEMENT}

This work is funded by the UK Engineering and Physical Sciences Research Council grant number EP/I032231/1.

\section{REFERENCES}

[1] M. A. Jensen, and Y. Rahmat-samii, "EM Interaction of Handset Antennas and a Human in Personal Communications," Proceedings of the IEEE, vol. 83, pp. 7-17, 1995.

[2] CTIA Certification, "Test Plan for Mobile Station Over the Air Performance”, v3.1, January 2011.

[3] L. Cung-Huan, E. Ofli, N. Chavannes, and N. Kuster, "Effects of Hand Phantom on Mobile Phone Antenna Performance," Antennas and Propagation, IEEE Transactions on, vol.57, no.9, pp.2763-2770, Sept. 2009.

[4] P. Hui, "Positive hand effects on mobile handset antennas," Microwave Conference, 2008. APMC 2008. Asia-Pacific , pp.1-4, 16-20 Dec. 2008. 